\title{
Epidural Hematoma after the Use of Subcutaneous Unfractionated Heparin and History of Epidural Tumor
}

\author{
Basem A. Abdelfattah, Troy Buck, Scott Byram \\ Department of Anesthesiology, Loyola University Medical Center, Maywood, IL, USA \\ Email: babdelfattah@Lumc.edu
}

Received 30 May 2014; revised 30 June 2014; accepted 15 July 2014

Copyright (C) 2014 by authors and Scientific Research Publishing Inc.

This work is licensed under the Creative Commons Attribution International License (CC BY). http://creativecommons.org/licenses/by/4.0/

(c) (i) Open Access

\section{Abstract}

The patient was a 66-year-old male with a history of renal cell carcinoma with metastasis to the L2, L3, and L4 vertebral bodies scheduled for a radical nephrectomy and adrenalectomy. Prior to surgery the patient had undergone 10 radiation treatments for the vertebral metastasis. Patient medications included colace, prednisone, ibuprofen (taken 4 days prior to procedure), sunitinib, hydrocodone, benazepril, nexium, rosuvastatin, allopurinol, and azor. Physical exam prior to surgery was normal with no focal findings. Laboratory values were within normal limits with a platelet count of 286. General anesthesia was planned with a pre-operative thoracic epidural for postoperative analgesia. The epidural was placed at the T9-T10 level without complication. Adequate pain control was present post operatively and the epidural was discontinued on post-operative day 3. At that time the patient was noted to have numbness over the bilateral lower extremities and decreased strength. Heparin 5000 units subcutaneous had been given 12 hours prior to discontinuation of the epidural. The patient was afebrile, hemoglobin was 7 , white blood cell count was 9.7, and platelets were 166 at time of epidural removal. Subcutaneous heparin was restarted 6 hours after catheter removal. The anesthesia acute pain service was contacted by the primary service 25 hours after discontinuation of the epidural catheter regarding complaints of persistent bilateral lower extremity weakness and sensory loss. An magnetic resonance image (MRI) revealed a focus posterior to the spinal cord at the T10/T11 interspace likely representing a hematoma. A high intensity T2 signal within the central spinal cord at T10/T11 was also observed; likely a vascular infarct. The patient was immediately scheduled for a T9-T10 laminectomy and evacuation of the epidural hematoma. No significant abnormalities were noted in coagulation studies prior to surgery. The patient was discharged on post-operative day 20 with no neurologic deficits. An epidural hematoma is rare with an estimated occurrence of $<1$ in 150,000 [1]. Issues related to anticoagulation therapy are involved in $25 \%-30 \%$ of cases. The utilization of three times daily dosed (TID) heparin could have played a role in the development of this complication in the present case. other contributing factors may have been chemotherapy and radiation therapy. These treatments 
are quite damaging to bone marrow and may cause severe marrow suppression thereby suppressing the function and number of platelets. Cancer cells are also capable of producing local cell signals which can initiate new blood vessel growth and proliferation [2]. This can also lead to blood vessels that are defective and leaky at the level of the endothelium. Increasing the number of fragile blood vessels may easily predispose this patient to laceration and shearing of blood vessels during epidural placement.

\section{Keywords}

Epidural Hematoma, Epidural Metastasis, Heparin

\section{Introduction}

This case addresses the occurrence of an epidural hematomain a 66-year-old male with a history of renal cell carcinoma with three times daily dosed subcutaneous unfractionated heparin (UFH) and concomitant epidural metastasis. Per the new American Society of Regional Anesthesia (ASRA) consensus guidelines on the management of neuraxial anesthesia, the safety in patients receiving doses greater than 10,000 units of UFH daily or more than twice daily dosing of UFH has not been established. In the case of epidural metastasis, chemotherapy and radiation therapy can lead to a hypocoagulable state. There is also the reminder for vigilance in the setting of any neurological deficits status post epidural anesthesia.

\section{Case Report}

A 66-year-old male with metastatic renal cell carcinoma (metastasis to the L2, L3, and L4 vertebral bodies) was scheduled for a radical nephrectomy and adrenalectomy. Prior to surgery the patient had undergone a total of ten radiation treatments for the lumbar vertebral metastasis. The last treatment occurred one weak prior to surgery. The patient's past medical history included hypertension, hyperlipidemia, and coronary artery disease. Past surgical history included mitral valve repair approximately 10 years prior and laser retina surgery 3 years prior. Medications at the time of surgery were docusate, prednisone, ibuprofen (last taken 4 days prior to surgery), sunitinib, hydrocodone with acetaminophen, benazepril, esomeprazole, rosuvastatin, allopurinol, and amlodipine/ olmesartan. Physical exam prior to surgery was unremarkable. Both motor and neurological exams were without focal findings. Laboratory values were within normal limits with a platelet count of 286.

General anesthesia with placement of pre-operative thoracic epidural for post-operative analgesia was planned for surgery. The epidural was placed using sterile technique at the T9-T10 level using a paramedian approach. Loss of resistance was noted at 7 centimeters and the catheter was taped at 12 centimeters. There were no complications encountered throughout placement of the epidural. Two large bore IV's, and an arterial line were placed. Anesthesia was induced and maintained utilizing propofol, lidocaine, sevoflurane, fentanyl, versed, and vecuronium. The surgery was noted to have 2 liters of blood loss with, 10 liters of crystalloid, 4 units of packed red blood cells, and 1 liter of colloid given. Urine output was 850 ccs. The patient was extubated at the conclusion of the case without complication. Patient noted right shoulder pain and right flank pain periumbilically adjacent to the incision. Shoulder pain was assessed and noted to be associated with positioning. The patient was mildly hypotensive in the PACU with systolic blood pressures ranging from 80 - 87. Post-operative hemoglobin was $7.5 \mathrm{mg} / \mathrm{dl}$ and patient was given an additional unit of packed red blood cells. Upon evaluation of the epidural, a level of T6 to L1 on left and T8 to T 11 on right was recorded. The epidural infusion was morphine 0.05 $\mathrm{mg} / \mathrm{ml}$ with bupivacaine $1 \mathrm{mg} / \mathrm{ml}$ in normal saline. Epidural settings were bolus $3 \mathrm{cc} / \mathrm{hr}$, rate $5 \mathrm{cc} / \mathrm{hr}$, lockout 20 min, with a maximum of $14 \mathrm{cc} / \mathrm{hr}$.

Following surgery the patient was followed daily by the acute pain service. The epidural was discontinued on post-operative day 3 at which time the patient was noted to have numbness and decreased strength in the bilateral lower extremities, which was attributed to local anesthetic motor blockade. Subcutaneous heparin (5000 units TID) had been given with the last dose 12 hours prior to discontinuation of the epidural. The patient was afebrile with a hemoglobin of 7, white blood cell count of 9.7, and platelets of 166 at the time the epidural was removed. No additional coagulation studies were performed. The epidural catheter tip was intact and the epidural site was 
without any signs of erythema or swelling. Subcutaneous heparin was not restarted until 6 hours after catheter removal. The anesthesia acute pain service was contacted by the primary surgical service 25 hours after discontinuation of the epidural catheter regarding complaints of persistent bilateral lower extremity weakness. Physical exam demonstrated the epidural site to be clean, dry, and with no induration or erythema. Motor weakness $3 / 5$ to plantar flexion and foot inversion/eversion were noted. The patient had sensory loss to pinprick in the S1 nerve distribution. Neurosurgery and the orthopedic spine service were consulted for evaluation of apossible epidural hematoma or abscess. An MRI done 5 hours after the initial consult demonstrated afocal collection posterior to the spinal cord at the T10/T11 interspace likely representing a hematoma as well as a high intensity T2 signal within the central spinal cord at T10/T11 likely representing a vascular infarct. The patient was immediately scheduled for a T9-T10 laminectomy with evacuation of the epidural hematoma. Coagulations studies were done prior to surgery showing an INR of 1.4, prothrombin time of 14, and partial thromboplastin time (aPTT) of 40.4. Two units of packed red blood cell's were transfused and the patient was given 10mg of IV decadronon the way to the operating room. A T9-10 posterior laminectomy was done and an epidural hematoma under pressure was discovered and evacuated without complication. The patient was transferred intubated to the neurological intensive care unit. The next morning a neurology exam demonstrated 5/5 strength of the bilateral upper limbs. There was marked paraparesis of the lower extremities with bilateral external rotation of both feet, and minimal movements (flexion, extension, inversion, eversion) of his feet. Bilateral patellar and ankle areflexia were noted. Patient, however, appeared to react to pinprick throughout. On post-operative day 2, significant improvement of the bilaterally lower extremities were seen with 4/5 muscle strength throughout. Patient was continued on decadron, hemoglobin of 9 was maintained for cord perfusion, and inpatient rehab was started on post-operative day 3. The patient was discharged on post-operative day 20, able to ambulate independently, and scheduled for outpatient rehab.

\section{Discussion}

A spinal hematoma is a collection of blood that occurs between the dura mater and the spine. Hemorrhage into the spinal canal most commonly occurs in the epidural space because of the prominent epidural venous plexus. It is a rare but potentially devastating complication of spinal and epidural anesthesia with an incidence of $<1$ in 150,000 [2]. Approximately one quarter to one third of all cases are associated with anticoagulation therapy [2]. The usual presentations are numbness and weakness of the lower extremities, or back pain that progresses toward paraparesis or quadriparesis. Early detection is critical, specifically within 8 hours of symptom onset, to increase the chance for adequate neural recovery. It is more likely to occur in anti-coagulated or thrombocytopenic patients, or in those with liver disease or alcoholism. Patients having taken non-steroidal anti-inflammatory drugs with antiplatelet effects or subcutaneous unfractionated heparin for deep vein thrombosis prophylaxis are viewed as having little risk for a spinal hematoma.

This patient received subcutaneous heparin 5000 U TID with the last dose given 12 hours prior to removal of the epidural catheter. ASRA guidelines state the safety of neuraxial blockade in patients receiving doses greater than 10,000 U of UFH daily or more than twice daily dosing of UFH has not been established. Although the use of thrice-daily UFH may lead to an increased risk of surgical-related bleeding, it is unclear whether there is an increased risk of spinal hematoma [3]. There are 9 published series totaling over 9000 patients who have received this therapy without complications. There are only 4 case reports of neuraxial hematomas, 3 epidural and 1 subarachnoid, during neuraxial block with the use of SC heparin [2]. ASRA guidelines do not have any specific recommendations for patients receiving subcutaneous heparin.

Another significant factor in this patient's past medical history is that of an epidural tumor. Tumors can predispose a patient to a hypercoagulable state through prothrombotic factors including the ability of tumor cells to produce and secrete procoagulant substances and inflammatory cytokines, however, chemotherapy and radiation therapy can lead to a hypocoagulable state. Chemotherapy and radiation therapy are quite damaging to bone marrow and may cause severe marrow suppression thereby suppressing the function and number of platelets, despite normal perioperative platelet count, prothrombin time, and thrombin time [4]. As a result our patient may still have had an underlying coagulopathy. Cancer cells are also capable of producing local cell signals which can initiate new blood vessel growth and proliferation [5]. The angiogenic cascade during tumor development consists of the release of angiogenic factors, binding of angiogenic factors to receptors on endothelial cells, endothelial cell activation, degradation of the basement membrane by proteases, and migration and proliferation of endothelial cells [6]. This can also lead to blood vessels that are defective and leaky at the level of the 
endothelium. Increasing the number of fragile blood vessels may easily predispose this patient to laceration and shearing of blood vessels during epidural placement.

One concern in this case was the lack of careful follow through by the anesthetic team. Though spinal hematoma's typically present with severe back pain (which this patient did not have), the neurologic deficits at the time of epidural discontinuation should still have been evaluated with frequent follow ups and neurological checks until resolution of deficits. This is especially prudent in the setting of TID dosed subcutaneous heparin. This patient had no imaging ordered till 25 hours after discontinuation of the epidural catheter.

Whether or not an epidural should have been placed is also an area of debate. In the setting of a patient with metastatic renal cell carcinoma, we believe it was appropriate for palliative care in this patient.

This case not only questions the safety of placing an epidural in patients being dosed with TID subcutaneous heparin, but also those with epidural metastasis. Though there may have been adequate timing between administrations of subcutaneous heparin, ASRA guidelines have not made absolute recommendations in the setting of TID dosed heparin. Also there are no specific guidelines regarding placement in the patient with epidural metastasis. Though this epidural was consciously placed at the T9-T10 level so as to avoid the area of noted prior metastasis, the risk of angiogenesis and vessel fragility in this patient may have still placed him at an increased risk for an epidural hematoma.

\section{Literature Review}

A literature review on this specific case did not yield many similar cases. A case submitted by Sandhu, H. et al. described a patient developing an epidural hematoma while on twice daily dosed UFH and a history of spinal stenosis [7].

\section{References}

[1] Dickman, C.A., Shedd, S.A., Spetzler, R.F., et al. (1990) Spinal Epidural Hematoma Associated with Epidural Anesthesia: Complications of Systemic Heparinization in Patients Receiving Peripheral Vascular Thrombolytic Therapy. Anesthesiology, 72, 947-950. http://dx.doi.org/10.1097/00000542-199005000-00028

[2] Barash, P., Cullen, B.F., Stoelting, R.K., Cahalan, M. and Stock, M.C. (2009) Clinical Anesthesia. 6th Edition, Epidural and Spinal Anesthesia, Lippincott Williams \& Wilkins, Philadelphia, 950-951.

[3] Horlocker, T.T., Wedel, D.J. and Benzon, H. (2010) Regional Anesthesia in the Anticoagulated Patient: Defining the Risks (the Second ASRA Consensus Conference on Neuraxial Anesthesia and Anticoagulation). Regional Anesthesia and Pain Medicine, 28, 172-197.

[4] Gordon, M.S., Mendelson, D.S. and Kato, G. (2010) Tumor Angiogenesis and Novel Antiangiogenic Strategies. International Journal of Cancer, 126, 1777-1787.

[5] Folkman J. (1971) Tumor Angiogenesis: Therapeutic Implications. The New England Journal of Medicine, 285, 11821186. http://dx.doi.org/10.1056/NEJM197111182852108

[6] Gupta, S.C., Kim, J.H., Prasad, S. and Aggarwal, B.B. (2010) Regulation of Survival, Proliferation, Invasion, Angiogenesis, and Metastasis of Tumor Cells through Modulation of Inflammatory Pathways by Nutraceuticals. Cancer and Metastasis Reviews, 29, 405-434. http://dx.doi.org/10.1007/s10555-010-9235-2

[7] Sandhu, H., Morley-Forster, P. and Spadafora, S. (2000) Epiduralhematoma Following Epidural Analgesia in a Patient Receiving Unfractionated Heparin for Thromboprophylaxis. Regional Anesthesia and Pain Medicine, 25, 72-75. 
Scientific Research Publishing (SCIRP) is one of the largest Open Access journal publishers. It is currently publishing more than 200 open access, online, peer-reviewed journals covering a wide range of academic disciplines. SCIRP serves the worldwide academic communities and contributes to the progress and application of science with its publication.

Other selected journals from SCIRP are listed as below. Submit your manuscript to us via either submit@scirp.org or Online Submission Portal.
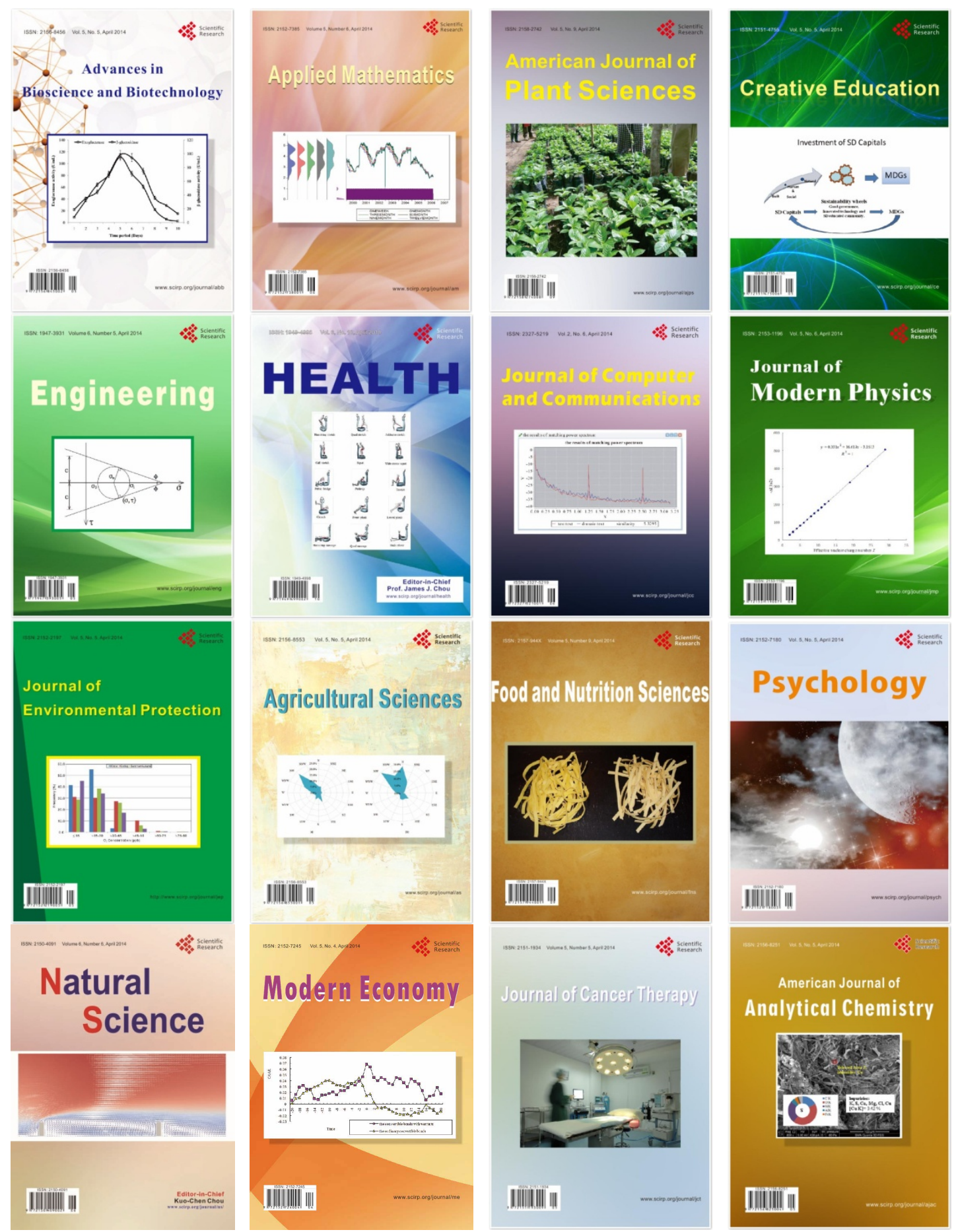